\title{
The Roles of Kinases in Familial Parkinson's Disease
}

\author{
Mark R. Cookson, ${ }^{1}$ William Dauer, ${ }^{2}$ Ted Dawson, ${ }^{3}$ Edward A. Fon, ${ }^{4}$ Ming Guo, ${ }^{5}$ and Jie Shen ${ }^{6}$ \\ ${ }^{1}$ Cell Biology and Gene Expression Unit, Laboratory of Neurogenetics, National Institute on Aging, Bethesda, Maryland 20892-3707, ${ }^{2}$ Departments of \\ Neurology and Pharmacology, Columbia University, New York, New York 10032, ${ }^{3}$ The Institute for Cell Engineering, Johns Hopkins University School of \\ Medicine, Baltimore, Maryland 21205, ${ }^{4}$ Center for Neuronal Survival, Montreal Neurological Institute, McGill University, Montreal, Quebec, Canada H3A \\ 2B4, ${ }^{5}$ Department of Neurology, Brain Research Institute, University of California, Los Angeles School of Medicine, Los Angeles, California 90095, and \\ ${ }^{6}$ Center for Neurologic Diseases, Brigham and Women's Hospital, Harvard Medical School, Boston, Massachusetts 02115
}

The purpose of this mini-symposium is to discuss some of the inherited forms of Parkinson's disease (PD) in view of recent data suggesting that some of the proteins affect cellular signaling pathways. As an illustration, we shall focus on two different kinases associated with recessive and dominant forms of PD. Mutations in the mitochondrial kinase PTEN (phosphatase and tensin homolog)induced kinase 1 (PINK1) are loss-of-function mutations in a normally neuroprotective protein. Loss-of-function mutations in model organisms have variable effects, from dramatic muscle and spermatid defects in Drosophila to more subtle neurophysiological abnormalities in mice. Several lines of evidence relate these to the action of a second gene for familial PD, parkin, an E3 ubiquitin ligase shown recently to have effects on Akt signaling. Mutations in leucine-rich repeat kinase 2 (LRRK2), a cytosolic kinase, are dominant and have the opposite effect of causing neuronal damage. The mechanism(s) involved are uncertain at this time because LRRK2 is a large and complex molecule with several domains. Increased kinase activity accounts for the action of at least some of the mutations, suggesting that hyperactive or misregulated kinase activity may lead to the damaging effects of LRRK2 in neurons. For both PINK1 and LRRK2, the following key question that needs to be answered: what are the physiological substrates that mediate effects in cells? Here, we will discuss some of the recent thinking about physiological and pathological roles for signaling in PD and how these may have therapeutic implications for the future.

Key words: Parkinson's disease; dopaminergic; genetics; human; knock-out mice; mitochondria; neuron death; phosphorylation; protein kinases; signal transduction

\begin{abstract}
Introduction
Parkinson's disease (PD) affects $>1$ million people in North America alone. PD is characterized by motor and cognitive dysfunction reflecting widespread neurodegeneration, especially of midbrain dopaminergic neurons. Although PD is typically a sporadic illness, there is growing recognition that genetic susceptibility plays an important role. Indeed, the discovery of mutations underlying rare inherited forms of PD has shed light onto the molecular mechanisms that contribute to the sporadic disease.

The purpose of this article, linked to a Society for Neuroscience mini-symposium, is to review recent insights into the function of two PD genes, PTEN (phosphatase and tensin homolog)-induced kinase 1 (PINK1) and leucine-rich repeat kinase 2 (LRRK2), and to illustrate how the study of these genes is beginning to uncover signaling events underlying PD-related neurodegeneration.
\end{abstract}

Pink1, parkin, and pathways to recessive parkinsonism Recessively inherited PINK1 mutations are a relatively frequent cause of parkinsonism (Valente et al., 2004). PINK1-related dis-

\footnotetext{
Received Aug. 14, 2007; accepted Aug. 20, 2007.

Correspondence should be addressed to Dr. Mark Cookson, Building 35/Porter Building, 1A116, 35 Convent Drive, Bethesda, MD 20892. E-mail: cookson@mail.nih.gov.

DOI:10.1523/JNEUROSCI.3695-07.2007

Copyright $\odot 2007$ Society for Neuroscience 0270-6474/07/2711865-04\$15.00/0
}

ease is clinically similar to idiopathic PD and responds well to dopamine replacement therapies. PINK1 contains a serine/threonine protein kinase domain (Valente et al., 2004), preceded by an N-terminal mitochondrial targeting sequence that localizes the protein to mitochondria. Mutations are found throughout the protein, although missense mutations are commonly found in the kinase region. Both truncating and destabilizing mutations are also found, which support the idea that pathogenic mutations cause disease through a loss of function. In addition, PINK1 overexpression protects against oxidative and apoptotic stressors in a kinase-dependent manner (Petit et al., 2005). Thus, PINK1 lossof-function appears to promote PD-related neurodegeneration.

One of the problems with testing whether PINK1 mutations are simple loss-of-function mutations is that knowledge of direct substrates is limited. Therefore, most reports to date have used autophosphorylation or generic substrates to measure enzyme activity. A limitation of these assays is that they may not fully capture all the ways in which mutations work; for example, if a mutation on the protein surface disrupted docking of the enzyme to a pathological substrate, then this might not be captured in an autophosphorylation mode. In part because of this limitation, but more importantly because substrates are probably key to understanding pathogenesis, the search for PINK1 substrates is a critical step for the field. Recently, a mitochondrial chaperone, tumor necrosis factor receptor-associated protein 1, has been 
shown to be phosphorylated by PINK1 in vitro and in cell models (Pridgeon et al., 2007). Additional confirmation that this substrate contributes to PINK1 pathogenesis in vivo is awaited, perhaps in some of the models recently described for PINK1 deficiency.

Studies of PINK1 in vivo support the notion that it functions at mitochondria. Loss-of-function of the Drosophila PINK1 homolog (Clark et al., 2006; Park et al., 2006; Yang et al., 2006) leads to morphological abnormalities of mitochondria and apoptosis in testes and flight muscles. Some, but not all, of these studies also report dopaminergic cell loss. These findings were similar to loss of function of the Drosophila parkin homolog, leading investigators to explore a potential link between these two PD genes. Indeed, parkin overexpression rescues the PINK1 phenotype, but PINK1 overexpression does not rescue parkin loss of function. These findings suggest a functional relationship between PINK1 and parkin and indicate that PINK1 functions upstream of parkin. A similar relationship awaits demonstration in mammalian systems. PINK1 knock-out (KO) (Kitada et al., 2007) mice exhibit abnormalities of evoked dopamine release but show no evidence of neurodegeneration. Electron microscopy analysis revealed that the density and morphology of mitochondria in the striatum of $P I N K 1^{-/-}$and control mice is similar (T. Kitada and J. Shen, unpublished results). These results indicate that, in contrast to Drosophila mutants, loss of PINK1 function in the mouse brain does not cause severe mitochondrial morphological defects. The reason for this apparent discrepancy is not clear and is the subject of active discussion.

Like PINK1, parkin mutations cause autosomal recessive parkinsonism (Kitada et al., 1998) and account for a large fraction of familial early onset cases (Lucking et al., 2000). Parkin is an E3 ubiquitin (Ub) ligase, and many PD-associated mutations likely lead to a loss of parkin Ub-ligase activity. This would be expected to cause target substrate accumulation, because E3 ubiquitination (via lysine 48 , or "K48" of ubiquitin) is required for proteasomal degradation. However, not all mutations inactivate the ubiquitination activity of parkin (Hampe et al., 2006; Matsuda et al., 2006), and most candidate parkin substrates do not accumulate in the brains of parkin KO mice (Goldberg et al., 2003; Itier et al., 2003; Von Coelln et al., 2004; Ko et al., 2005; Perez and Palmiter, 2005; Periquet et al., 2005). Thus, a number of key questions remain about the pathogenic mechanisms related to parkin and whether proteasomal degradation is indeed the critical function.

Ubiquitination has been implicated in other cellular functions (Hicke and Dunn, 2003; Mukhopadhyay and Riezman, 2007), including signaling and protein trafficking. This typically involves mono-, multi-mono, or K63-linked-Ub conjugation (Hampe et al., 2006; Matsuda et al., 2006), and parkin is capable of catalyzing such linkages. For example, parkin monoubiquitinates epidermal growth factor (EGF) receptor pathway substrate 15 (Eps15), a Ub-binding adaptor protein involved in the endocytosis and trafficking of the EGF receptor (EGFR), a receptor tyrosine kinase. Ubiquitination of Eps15 may interfere with the ability of the Eps 15 to bind ubiquitinated EGFR, thereby delaying EGFR internalization and degradation and promoting prosurvival phosphatidylinositol 3 kinase-Akt signaling. Parkin also monoubiquitinates protein interacting with C-kinase 1 (PICK1) (Joch et al., 2007), an adaptor protein involved in the trafficking of neurotransmitter receptors, transporters, and ion channels (Madsen et al., 2005). In contrast to other E3 Ub-ligases, which ubiquitinate cell surface receptors and channels, the action of parkin on Eps15 and PICK1 indicate that it affects kinase signal- ing pathways by ubiquitinating downstream adaptor proteins involved in endocytosis and trafficking. Such a role for parkin may explain the lack of accumulation of most known parkin substrates in parkin null mice and the potential functional link between parkin and PINK1.

\section{LRRK2, a kinase for dominant PD}

Dominant mutations of LRRK2 are the most common cause of inherited PD (Paisan-Ruiz et al., 2004; Zimprich et al., 2004; Bonifati, 2007), and most cases of LRRK2-related PD are clinically and pathologically indistinguishable from the idiopathic disease. LRRK2 contains both GTPase and kinase domains, as well as two protein-protein interactions domains (leucine-rich and WD40 repeats). Definitively pathogenic mutations have been identified in the GTPase and kinase domains, as well as the region between these domains.

Significant efforts have been made to determine whether PD mutations alter LRRK2 kinase activity. There is consensus that G2019S significantly increases LRRK2 kinase function in assays of either autophosphorylation or phosphorylation of generic substrates (West et al., 2005; Greggio et al., 2006; MacLeod et al., 2006; Hatano et al., 2007; Jaleel et al., 2007; Luzon-Toro et al., 2007). However, controversy exists regarding whether the other PD mutations alter LRRK2 kinase function (Gloeckner et al., 2006; Jaleel et al., 2007). Mutations in the GTP-binding domain diminish the (admittedly low) rate of GTP hydrolysis seen with wild-type LRRK2 (Lewis et al., 2007; Li et al., 2007), suggesting that these mutations may not affect kinase activity per se but how kinase activity is regulated by GTP binding. Clarifying the effects of PD mutation on kinase function awaits the identification of true LRRK2 substrates, because autophosphorylation or model substrate assays may not accurately reflect LRRK2 function.

Multiple LRRK2 PD mutants show enhanced toxicity, causing significantly greater cell death than the wild-type protein in cell lines and primary neurons. Notably, abolishing LRRK2 kinase function diminishes the toxicity of all PD mutants (Greggio et al., 2006; Smith et al., 2006) although most of these mutations do not appear to enhance kinase function. Thus, most PD mutations appear to cause cell death by altering some other feature of LRRK2 biology but that nonetheless requires intact (basal) kinase function. The logical connection is that LRRK2 is a signaling molecule and that kinase activity is one key part of the signaling process. Presumably, LRRK2 becomes pathogenic when the kinase is hyperactive or misregulated, and this may involve signaling pathways. Some recent evidence suggests that LRRK2 or homologs in other species have roles in neurite outgrowth and sorting of molecules along axons (MacLeod et al., 2006; Sakaguchi-Nakashima et al., 2007). Therefore, LRRK2 probably has activities that are important (perhaps even required) for normal neuronal function. Understanding the relationship between pathological and normal signaling is clearly the key step for the field: is it the same set of signals but misregulated or novel pathways only accessed by the mutant proteins?

As an example of a novel property of mutant LRRK2, some mutations dramatically alter the subcellular distribution of the protein, causing it to concentrate in string-like filamentous structures rather than its normal diffuse cytosolic pattern (C. C.-Y. Ho and H. Rideout, unpublished observations). Notably, blocking kinase activity virtually abolishes filament formation, linking LRRK2 filament formation to neurotoxicity. In previously identified cases, filament formation reflects a homotypic proteinprotein interaction that mediates signaling, and oligomerization promotes the recruitment of signaling molecules into spatially 
defined complexes that facilitates their interaction and activation. LRRK2 oligomerizes and filament-forming mutations enhance its oligomeric state (Dauer, Ho, and Rideout, unpublished observations). LRRK2 oligomerization appears to generate a protein scaffold that recruits other signaling molecules. Filamentforming mutations may act by leading to a structural change in LRRK2 that exposes an autophosphorylation site and that blocking kinase function prevents filament formation (and therefore substrate recruitment and toxicity) by preventing the formation of the phosphomotif required for oligomerization. It is of interest that a kinase-dependent accumulation of LRRK2 has also been proposed for other mutations both in vitro (Greggio et al., 2006) and in vivo (MacLeod et al., 2006), although not all studies have reported increased inclusion bodies (West et al., 2005), suggesting that experimental details such as choice of mutation and expression levels may be important. These data suggest a novel signaling mechanism for LRRK2 and highlight the notion that blocking LRRK2 self-association may be a novel therapeutic strategy for PD.

\section{Summary}

The identification PD genes have provided an array of new tools with which to unravel PD pathogenesis. PINK1 and parkin appear functionally related and to be involved in neuroprotective signaling, although the mechanistic aspects are poorly understood. LRRK2 mutations appear to cause a toxic gain of function that requires intact kinase function. The fact that all of these proteins contain well understood catalytic motifs is enabling mechanistic studies, and the identification of bona fide substrates should greatly enhance our understanding of the detailed relationship between genes and neuronal survival.

\section{References}

Bonifati V (2007) LRRK2 low-penetrance mutations (Gly2019Ser) and risk alleles (Gly2385Arg)-linking familial and sporadic Parkinson's disease. Neurochem Res 32:1700-1708.

Clark IE, Dodson MW, Jiang C, Cao JH, Huh JR, Seol JH, Yoo SJ, Hay BA, Guo M (2006) Drosophila pink1 is required for mitochondrial function and interacts genetically with parkin. Nature 441:1162-1166.

Gloeckner CJ, Kinkl N, Schumacher A, Braun RJ, O'Neill E, Meitinger T, Kolch W, Prokisch H, Ueffing M (2006) The Parkinson disease causing LRRK2 mutation I2020T is associated with increased kinase activity. Hum Mol Genet 15:223-232.

Goldberg MS, Fleming SM, Palacino JJ, Cepeda C, Lam HA, Bhatnagar A, Meloni EG, Wu N, Ackerson LC, Klapstein GJ, Gajendiran M, Roth BL, Chesselet MF, Maidment NT, Levine MS, Shen J (2003) Parkindeficient mice exhibit nigrostriatal deficits but not loss of dopaminergic neurons. J Biol Chem 278:43628-43635.

Greggio E, Jain S, Kingsbury A, Bandopadhyay R, Lewis P, Kaganovich A, van der Brug MP, Beilina A, Blackinton J, Thomas KJ, Ahmad R, Miller DW, Kesavapany S, Singleton A, Lees A, Harvey RJ, Harvey K, Cookson MR (2006) Kinase activity is required for the toxic effects of mutant LRRK2/ dardarin. Neurobiol Dis 23:329-341.

Hampe C, Ardila-Osorio H, Fournier M, Brice A, Corti O (2006) Biochemical analysis of Parkinson's disease-causing variants of Parkin, an E3 ubiquitin-protein ligase with monoubiquitylation capacity. Hum Mol Genet 15:2059-2075.

Hatano T, Kubo S, Imai S, Maeda M, Ishikawa K, Mizuno Y, Hattori N (2007) Leucine-rich repeat kinase 2 associates with lipid rafts. Hum Mol Genet 16:678-690.

Hicke L, Dunn R (2003) Regulation of membrane protein transport by ubiquitin and ubiquitin-binding proteins. Annu Rev Cell Dev Biol 19:141-172.

Itier JM, Ibanez P, Mena MA, Abbas N, Cohen-Salmon C, Bohme GA, Laville M, Pratt J, Corti O, Pradier L, Ret G, Joubert C, Periquet M, Araujo F, Negroni J, Casarejos MJ, Canals S, Solano R, Serrano A, Gallego E, et al. (2003) Parkin gene inactivation alters behaviour and dopamine neurotransmission in the mouse. Hum Mol Genet 12:2277-2291.
Jaleel M, Nichols RJ, Deak M, Campbell DG, Gillardon F, Knebel A, Alessi DR (2007) LRRK2 phosphorylates moesin at threonine-558: characterization of how Parkinson's disease mutants affect kinase activity. Biochem J 405:307-317.

Joch M, Ase AR, Chen CX, Macdonald PA, Kontogiannea M, Corera AT, Brice A, Seguela P, Fon EA (2007) Parkin-mediated monoubiquitination of the PDZ protein PICK1 regulates the activity of acid-sensing ion channels. Mol Biol Cell 18:3105-3118.

Kitada T, Asakawa S, Hattori N, Matsumine H, Yamamura Y, Minoshima S, Yokochi M, Mizuno Y, Shimizu N (1998) Mutations in the parkin gene cause autosomal recessive juvenile parkinsonism. Nature 392:605-608.

Kitada T, Pisani A, Porter DR, Yamaguchi H, Tscherter A, Martella G, Bonsi P, Zhang C, Pothos EN, Shen J (2007) Impaired dopamine release and synaptic plasticity in the striatum of PINK1-deficient mice. Proc Natl Acad Sci USA 104:11441-11446.

Ko HS, von Coelln R, Sriram SR, Kim SW, Chung KK, Pletnikova O, Troncoso J, Johnson B, Saffary R, Goh EL, Song H, Park BJ, Kim MJ, Kim S, Dawson VL, Dawson TM (2005) Accumulation of the authentic parkin substrate aminoacyl-tRNA synthetase cofactor, p38/JTV-1, leads to catecholaminergic cell death. J Neurosci 25:7968-7978.

Lewis PA, Greggio E, Beilina A, Jain S, Baker A, Cookson MR (2007) The R1441C mutation of LRRK2 disrupts GTP hydrolysis. Biochem Biophys Res Commun 357:668-671.

Li X, Tan YC, Poulose S, Olanow CW, Huang XY, Yue Z (2007) Leucinerich repeat kinase 2 (LRRK2)/PARK8 possesses GTPase activity that is altered in familial Parkinson's disease R1441C/G mutants. J Neurochem 103:238-247.

Lucking CB, Durr A, Bonifati V, Vaughan J, De Michele G, Gasser T, Harhangi BS, Meco G, Denefle P, Wood NW, Agid Y, Brice A (2000) Association between early-onset Parkinson's disease and mutations in the parkin gene. French Parkinson's Disease Genetics Study Group. N Engl J Med 342:1560-1567.

Luzon-Toro B, de la Torre ER, Delgado A, Perez-Tur J, Hilfiker S (2007) Mechanistic insight into the dominant mode of the Parkinson's diseaseassociated G2019S LRRK2 mutation. Hum Mol Genet 16:2031-2039.

MacLeod D, Dowman J, Hammond R, Leete T, Inoue K, Abeliovich A (2006) The familial Parkinsonism gene LRRK2 regulates neurite process morphology. Neuron 52:587-593.

Madsen KL, Beuming T, Niv MY, Chang CW, Dev KK, Weinstein H, Gether U (2005) Molecular determinants for the complex binding specificity of the PDZ domain in PICK1. J Biol Chem 280:20539-20548.

Matsuda N, Kitami T, Suzuki T, Mizuno Y, Hattori N, Tanaka K (2006) Diverse effects of pathogenic mutations of Parkin that catalyze multiple monoubiquitylation in vitro. J Biol Chem 281:3204-3209.

Mukhopadhyay D, Riezman H (2007) Proteasome-independent functions of ubiquitin in endocytosis and signaling. Science 315:201-205.

Paisan-Ruiz C, Jain S, Evans EW, Gilks WP, Simon J, van der Brug M, Lopez de Munain A, Aparicio S, Gil AM, Khan N, Johnson J, Martinez JR, Nicholl D, Carrera IM, Pena AS, de Silva R, Lees A, Marti-Masso JF, Perez-Tur J, Wood NW, Singleton AB (2004) Cloning of the gene containing mutations that cause PARK8-linked Parkinson's disease. Neuron 44:595-600.

Park J, Lee SB, Lee S, Kim Y, Song S, Kim S, Bae E, Kim J, Shong M, Kim JM, Chung J (2006) Mitochondrial dysfunction in Drosophila PINK1 mutants is complemented by parkin. Nature 441:1157-1161.

Perez FA, Palmiter RD (2005) Parkin-deficient mice are not a robust model of parkinsonism. Proc Natl Acad Sci USA 102:2174-2179.

Periquet M, Corti O, Jacquier S, Brice A (2005) Proteomic analysis of parkin knockout mice: alterations in energy metabolism, protein handling and synaptic function. J Neurochem 95:1259-1276.

Petit A, Kawarai T, Paitel E, Sanjo N, Maj M, Scheid M, Chen F, Gu Y, Hasegawa H, Salehi-Rad S, Wang L, Rogaeva E, Fraser P, Robinson B, St George-Hyslop P, Tandon A (2005) Wild-type PINK1 prevents basal and induced neuronal apoptosis, a protective effect abrogated by Parkinson disease-related mutations. J Biol Chem 280:34025-34032.

Pridgeon JW, Olzmann JA, Chin LS, Li L (2007) PINK1 protects against oxidative stress by phosphorylating mitochondrial chaperone TRAP1. PLoS Biol 5:e172.

Sakaguchi-Nakashima A, Meir JY, Jin Y, Matsumoto K, Hisamoto N (2007) LRK-1, a C. elegans PARK8-related kinase, regulates axonal-dendritic polarity of SV proteins. Curr Biol 17:592-598. 
Smith WW, Pei Z, Jiang H, Dawson VL, Dawson TM, Ross CA (2006) Kinase activity of mutant LRRK2 mediates neuronal toxicity. Nat Neurosci 9:1231-1233.

Valente EM, Abou-Sleiman PM, Caputo V, Muqit MM, Harvey K, Gispert S, Ali Z, Del Turco D, Bentivoglio AR, Healy DG, Albanese A, Nussbaum R, Gonzalez-Maldonado R, Deller T, Salvi S, Cortelli P, Gilks WP, Latchman DS, Harvey RJ, Dallapiccola B, Auburger G, Wood NW (2004) Hereditary early-onset Parkinson's disease caused by mutations in PINK1. Science 304:1158-1160.

Von Coelln R, Thomas B, Savitt JM, Lim KL, Sasaki M, Hess EJ, Dawson VL, Dawson TM (2004) Loss of locus coeruleus neurons and reduced startle in parkin null mice. Proc Natl Acad Sci USA 101:10744-10749.

West AB, Moore DJ, Biskup S, Bugayenko A, Smith WW, Ross CA, Dawson
VL, Dawson TM (2005) Parkinson's disease-associated mutations in leucine-rich repeat kinase 2 augment kinase activity. Proc Natl Acad Sci USA 102:16842-16847.

Yang Y, Gehrke S, Imai Y, Huang Z, Ouyang Y, Wang JW, Yang L, Beal MF, Vogel H, Lu B (2006) Mitochondrial pathology and muscle and dopaminergic neuron degeneration caused by inactivation of Drosophila Pink1 is rescued by Parkin. Proc Natl Acad Sci USA 103:10793-10798.

Zimprich A, Biskup S, Leitner P, Lichtner P, Farrer M, Lincoln S, Kachergus J, Hulihan M, Uitti RJ, Calne DB, Stoessl AJ, Pfeiffer RF, Patenge N, Carbajal IC, Vieregge P, Asmus F, Muller-Myhsok B, Dickson DW, Meitinger T, Strom TM, Wszolek ZK, Gasser T (2004) Mutations in LRRK2 cause autosomal-dominant parkinsonism with pleomorphic pathology. Neuron 44:601-607. 